\title{
Principios de Neurociencia aplicados en la Educación Universitaria
}

\author{
Gabriel Valerio*, Jorge Jaramillo, Ricardo Caraza y Ruth Rodríguez \\ Tecnológico de Monterrey, Campus Monterrey, Ave. Eugenio Garza Sada 2501, Monterrey, N. L., México, \\ 64849 (e-mail: gvalerio@itesm.mx, jojaramillo@cantab.net, rcaraza@itesm.mx, ruthrdz@itesm.mx)
}

* Autor a quien debe ser dirigida la correspondencia

Recibido Oct. 6, 2015; Aceptado Dic. 7, 2015; Versión final Mar. 28, 2016, Publicado Ago. 2016

\begin{abstract}
Resumen
El principal objetivo del trabajo fue determinar si hay un aumento en la atención, la motivación y el desempeño académico de los estudiantes universitarios, cuando las sesiones de clases se llevan a cabo en un ambiente enriquecido en el uso de prácticas docentes basadas en los principios de las ciencias del cerebro. La investigación tuvo un diseño de metodología mixta. Se desarrolló un experimento con dos grupos de la misma asignatura y con el mismo profesor. En el grupo experimental se aplicaron las prácticas docentes ricas en uso de prácticas basadas en recomendaciones de la neurociencia, mientras el grupo control se impartió en un ambiente académico tradicional. Como herramientas de recolección de datos fueron utilizadas la observación no participante, dos pruebas estandarizadas y una encuesta semanal para medir los niveles de motivación. Los resultados mostraron que las tres variables dependientes analizadas (atención, motivación y desempeño académico) fueron superiores en el grupo experimental. Estos hallazgos invitan a la inclusión de los temas de neurociencia en la agenda de investigación educativa.
\end{abstract} cognitivos

\section{Principles of Neuroscience applied to University Education}

\begin{abstract}
The main objective of this work was to determine whether there is an increase in certain cognitive processes such as attention, motivation, and academic performance, for undergraduate university students, when the class sessions are conducted in an environment that is rich in teaching practices based on neuroscience principles. The research had a mixed methodology design. The experiment was developed with two groups of the same course and with the same professor. In the experimental group, the novel practices were implemented and in the control group, a traditional academic environment was structured. As data collection tools, non-participant observation, two standardized tests and a weekly survey to measure levels of motivation were used. The results showed that the three dependent variables analyzed (attention, motivation, and academic performance), had a positive impact in the experimental group. The academic performance of the students increased as well. These findings suggest the inclusion of neuroscience matters in the educational research agenda.
\end{abstract}

Keywords: neuroscience; cognitive psychology; education; teaching strategies; cognitive processes 


\section{INTRODUCCIÓN}

El creciente volumen de información y la tendencia de la hiper-especialización de las carreras universitarias, común en nuestra moderna sociedad del conocimiento, demanda la reestructuración de los procesos de enseñanza y aprendizaje de las instituciones y profesionales en el campo educativo (Dyson y Renk, 2006; Arnett, 2000). Estas innovaciones deben dar una solución no solo a estos requerimientos, sino también a la falta de atención y motivación de los estudiantes debido a la saturación actual de contenidos (Pulido, 2011; Beck et al., 2003). Dadas las exigencias, retos y situaciones cada vez más complejos, que afectan o impiden el curso natural de aprendizaje, el aumento significativo de los estudiantes con estrés (Putwain, 2007) y el poco interés entre ellos, muchas teorías y todo tipo de propuestas han surgido. En este sentido, y dentro de la gama de posibles soluciones, destaca el valor de las estrategias de enseñanza basadas en la neurociencia (Fischer y Immordino-Yang, 2008). Por esta razón, se diseñó y ejecutó un experimento para explorar la eficiencia de este tipo de implementaciones alrededor de la siguiente pregunta de investigación: ¿existe un aumento en los niveles de atención, motivación y rendimiento académico de los estudiantes universitarios, cuando las sesiones de clases se llevan a cabo utilizando prácticas de enseñanza basadas en los principios de la neurociencia?

Desde hace una década, aproximadamente, con el avance de las neurociencias ha existido un interés creciente en relacionar estos conocimientos con la educación, al tratar de entender cómo el cerebro cambia y se adapta durante el aprendizaje. En este sentido, Ansari et al., (2012) conjuntaron información sobre este campo, planteando cómo a través de diferentes herramientas se han estado investigando los circuitos cerebrales involucrados en diferentes habilidades académicas. Señalando, de forma particular, los estudios en neurociencias cognitivas donde se han obtenido avances para aumentar nuestro conocimiento sobre cómo contribuyen el cerebro y las funciones cognitivas al funcionamiento del aprendizaje. Dentro de estas funciones cognitivas que favorecen el aprendizaje, en este trabajo, nos hemos centrado en la atención y motivación, como base de las intervenciones para mejorar el rendimiento académico.

La atención es la encargada de realizar el proceso de selección de la información dentro del sistema nervioso, siendo el elemento fundamental que articula todos los procesos cognoscitivos, dirigiendo y seleccionando la información que se va a procesar. De acuerdo a Bruning et al. (2012), la atención se refiere a la asignación de recursos cognitivos que hace una persona frente a una tarea. Estudios previos sobre atención (Riccio et al., 1994) han mostrado que los seres humanos estamos muy limitados en el número de cosas a las que se pueden prestar atención al mismo tiempo. Dentro del proceso atencional, la atención selectiva, es decir la función cognitiva encargada de focalizarnos e ignorar la distracción, es necesaria en el proceso académico de aprendizaje (Stevens y Bavelier, 2012). Es un proceso que permite seleccionar y focalizar un estímulo en particular para un procesamiento posterior mientras se suprime información irrelevante o distractores. Cuando la atención se dirige hacia los atributos de un estímulo o hacia determinada información, se identifica un incremento en la actividad de las áreas corticales de asociación que procesan determinado estímulo o información (Gazzaley y Nobre, 2012; Booth et al., 2003).

Por otra parte, la motivación nos permite obtener gratificación al realizar alguna actividad, lo que incrementa la atención y el aprendizaje. El circuito responsable es el circuito de la recompensa, el cual consiste en una intrincada red de conexiones que involucran tanto estructuras subcorticales como prefrontales, siendo uno de sus principales centros el núcleo accumbens (Haber y Knutson, 2010). Al ir comprendiendo este circuito neuronal se ha ido identificando que no solo consiste en obtener gratificación sino que incluye más procesos. La función principal de la recompensa es inducir emociones positivas para que el organismo se aproxime, aumente la frecuencia de la conducta diana y evite la extinción del comportamiento. Kim (2013) propone cómo el modelo neurocientífico de la motivación tiene implicaciones en la educación y al estimularse puede incrementar la motivación por aprender. El nuevo conocimiento se debe presentar de forma interesante y novedosa, recompensando la participación. Después, este valor positivo del desempeño del alumno debe ser mantenido hasta que se adquiera un control cognitivo, creando una meta que sirva de incentivo para regular la motivación a lo largo del tiempo.

En el proceso de aprendizaje y adquisición de conocimientos, la memoria realiza un rol predominante, ya que no solo se trata de almacenar información, sino que su principal función es la de evocar la información aprendida sobre todo a largo plazo. Esta memoria a largo plazo generalmente se divide en declarativa y nodeclarativa. La memoria declarativa se divide a su vez en episódica, la relacionada con eventos o episodios y la semántica, relacionada con conocimientos, siendo esta memoria declarativa la que se relaciona con el rendimiento académico. Las teorías que buscan explicar las vías neuronales subyacentes a la memoria a largo plazo explican que la información registrada inicialmente en la neocorteza es integrada por el complejo hipocámpico y la cara medial del lóbulo temporal y estructuras relacionadas en el diencéfalo, con esto se forman los trazos de memoria a largo plazo (Daw y Shohamy, 2008; Moscovitch et al., 2006). 
La existencia de conocimiento previo al cual la nueva información puede ser relacionada, permite un mejor desempeño de la memoria. Asimismo, la información nueva, novedosa y cambiante engancha nuestro proceso atencional y motivacional, lo que permite la adquisición de nuevo conocimiento, mejora nuestra memoria (van Kesteren et al., 2012) y, con ello, el desempeño académico.

Al entender cómo funciona el cerebro, los profesores están mejor preparados para ayudar a los alumnos a aprender. La neurociencia propone algunas prácticas que pueden ser implementadas dentro del salón de clases. Por ejemplo, en cuanto a la atención, Willis (2009) y Jensen (2004) proponen el uso de pausas en los niveles de atención, ya que después de cada nueva experiencia de aprendizaje se necesita tiempo para "imprimir" el aprendizaje. Para la motivación, Willis (2009) afirma que la dopamina, además de reducir el estrés, propicia la perseverancia, la curiosidad y en general la sensación de placer. Según esta autora, reír, ayudar a alguien, expresar gratitud, ser optimista, escuchar música, bailar, hacer ejercicio, predecir y superar un reto, son actividades que ayudan a disparar la hormona del placer y muchas de estas actividades pueden ser practicadas en el salón de clases. En cuanto a la memoria, Bjork (mencionado en Sundem, 2012) asegura que si estudias y esperas un rato para repasar, es más fácil que el contenido se quede en tu memoria, por ello recomienda tomar notas inmediatamente después de la clase, en lugar que durante la clase. Asimismo, se recomiendan las repeticiones en distintos escenarios para facilitar la memorización duradera y activar un conocimiento ya almacenado que permita conectar el nuevo conocimiento (Bjork mencionado en Sundem, 2012; van Kesteren et al., 2012; Willis, 2006).

Los avances en la neurociencia han proporcionado nuevas perspectivas para el desarrollo de las funciones cognitivas. De acuerdo a Oliver (2011), esto es de gran interés para los educadores que se ocupan de cómo aprenden los estudiantes. Este trabajo pretende rescatar justamente los aportes de la neurociencia para mejorar el aprendizaje de los alumnos. Finalmente, una cuestión importante en el debate sobre la neurociencia educacional es la transferencia de pensamiento y hallazgos entre la neurociencia y la educación (van der Meulen et al., 2015), facilitando con ello un diálogo interdisciplinario constructivo.

En este trabajo se busca reconocer si hay diferencias en los niveles de atención, motivación y rendimiento académico de los estudiantes universitarios, cuando se hace uso de estrategias de enseñanza basadas en la neurociencia, en lugar de un entorno educativo tradicional.

\section{METODOLOGÍA}

Con el fin de responder a la pregunta de investigación central establecida, se consideró conveniente trabajar bajo un paradigma de investigación mixto. El objetivo primario fue comparar dos grupos de estudiantes en un solo experimento, centrándose principalmente en la atención y motivación, además del desempeño académico. Es importante resaltar que se realizaron pruebas para asegurarse que todos los alumnos tuvieran similares capacidades de atención y de memoria, es decir, que tuvieran la misma base de capacidades cognitivas. Con esto, se aseguró que los alumnos que participaron en el estudio no presentaban, por ejemplo, problemas de déficit de atención o de capacidad de memoria.

El test de homogeneidad número 1 (Test de Atención d2) se utilizó para medir la capacidad de atención de corto plazo y la velocidad de procesamiento. El test de homogeneidad número 2 (sub-test de memoria lógica del Test Barcelona), se utilizó para cuantificar la capacidad de memoria verbal, tanto inmediata como diferida. Una vez validada la atención y la capacidad de memoria, se utilizó una encuesta de percepción con el fin de cuantificar el nivel de motivación de los estudiantes. Esta encuesta se basa en seis factores: motivación intrínseca y de relevancia personal, eficiencia, libre determinación, motivación para la acción, la motivación para la calificación y la percepción general (Glynn et al., 2009). Por último, se utilizó un registro de calificaciones del estudiante como el indicador de rendimiento académico.

En la parte cualitativa, se utilizó la observación no participativa durante las sesiones de clase para medir la atención y la motivación. Dichos procesos cognitivos se evaluaron a través del análisis y el estudio del lenguaje corporal. Los investigadores trabajaron simultáneamente en los dos grupos durante la misma fase del proceso de investigación, manteniendo una independencia durante el análisis. Los resultados se integraron eventualmente para una interpretación concluyente. La Tabla 1 resume el diseño de las diferentes evaluaciones o herramientas de recolección de datos utilizados durante la ejecución del experimento.

La prueba d2 (Brickenkamp y Zillmer, 2002) se utiliza para conocer la capacidad de atención/concentración en una tarea determinada. Es una evaluación desarrollada por tiempo, en donde el objetivo principal es marcar las letras "d" que estén acompañadas de 2 rayitas. Se trabaja con 14 líneas, una línea a la vez. El administrador notifica al estudiante cuándo comienza el tiempo y al cabo de 20 segundas dice "¡Cambio!" para continuar con la siguiente línea inmediata. Esta prueba se aplicó al inicio y al final del periodo de 
experimentación y no se utilizaba para medir las variables de estudio, sino para asegurar que todos los participantes en el estudio tuvieran similares capacidades de atención.

Tabla 1: Resumen de las herramientas de recolección de datos.

\begin{tabular}{|l|l|}
\hline $\begin{array}{l}\text { Aseguramiento de la muestra: } \\
\text { Atención y Memoria }\end{array}$ & $\begin{array}{l}\text { Test de homogeneidad número 1 (d2) } \\
\text { Test de homogeneidad número 2 (sub-test de memoria) }\end{array}$ \\
\hline Atención & Observación no participativa \\
\hline Motivación & $\begin{array}{l}\text { Observación no participativa } \\
\text { Encuesta semanal de percepción motivacional (Glynn, Taasoobshirazi y } \\
\text { Brickman, 2009) }\end{array}$ \\
\hline Desempeño Académico & Registro de notas de la clase \\
\hline
\end{tabular}

El test de homogeneidad número 2 o sub-test de memoria lógica del "Test Barcelona" (Peña-Casanova et al., 1997) se realiza a través de una repetición inmediata y otra diferida (luego de aproximadamente 10 minutos) de dos textos previamente divulgados. Se establece además un listado de preguntas que corresponden a cada fragmento en los que está dividido el texto, con el objetivo de explorar diferencias entre la evocación libre y la evocación mediante claves. El administrador de la prueba informa a los alumnos que les leerá un par de historias, que deben prestar mucha atención y luego repetirlas lo más exactamente posible en el documento que éste les ha proporcionado. La calificación se realiza asignando un punto por cada fragmento de texto recordado; e indirectamente, un 1 punto a la pregunta correspondiente a ese fragmento. También se asigna 0.5 puntos si el fragmento es recordado incompletamente; o bien, si el alumno evoca tal fragmento sin citar las palabras concretas con que se le leyó: diciendo sinónimos, una idea general o una síntesis correcta de la información. La puntuación de cada etapa de la evaluación se obtiene sumando los puntos obtenidos por fragmentos. Los medios puntos se redondean por defecto. En la memoria diferida, en caso de que el estudiante no recuerde nada, se debe descontar un punto por cada ayuda que quién ejecute la prueba otorgue. La puntuación máxima a obtener por cada subconjunto es de 23 puntos, que posteriormente son equiparados con su respectivo percentil, con base en una tabla de asignaciones previamente determinada. Al igual que la prueba d2, se aplicó la misma al inicio y al final del periodo de experimentación y sirvió para asegurar que los participantes en el estudio tenían similares niveles de capacidad de memoria.

En lo que respecta a la observación, para medir la motivación y la atención, se utilizó un sistema de grabación integrado en el aula. Esto permitió grabar todas las sesiones de clases a lo largo de 8 semanas (24 horas de observación por grupo). En la Tabla 2 se presenta un extracto del instrumento que se usó para evaluar los niveles de atención y motivación de los estudiantes durante la clase en ambos grupos en base a los comportamientos observados. Cabe mencionar que dicha evaluación del comportamiento se basó en la revisión de trabajos sobre lenguaje corporal y lenguaje no verbal (Bruning, et al., 2012; Álava, 2004; Fast, 2002; Ribbens y Thompson, 2002).

Tabla 2: Extracto de la guía de observación no participativa.

\begin{tabular}{|l|l|l|}
\hline $\begin{array}{c}\text { Categoría de } \\
\text { comportamiento }\end{array}$ & \multicolumn{1}{|c|}{ Comportamiento observado } & $\begin{array}{c}\text { Número de } \\
\text { incidentes }\end{array}$ \\
\hline Atención A & Sostiene la cabeza con una mano y el dedo índice en la mejilla & \\
\hline Inatención F & Frecuentemente cruza los brazos & \\
\hline Motivación A & Imitación efusiva & \\
\hline Desmotivación F & Externaliza stress & \\
\hline
\end{tabular}

Un voluntario, ajeno al grupo principal de investigación, realizó el análisis de la observación no participativa. Este observador recibió un formulario de registro y luego revisó cada uno de los videos generados durante todo el experimento. Este formulario registra comportamientos asociados con la atención y la motivación para luego funcionar como una base de datos para normalizar y definir los niveles de los procesos cognitivos. El observador no conocía el experimento en sí, ni la naturaleza o la diferencia de cualquier grupo de muestra. Esto se hizo con el fin de reducir el sesgo de la aplicación.

Las encuestas para medir la motivación (Glynn et al., 2009) fueron administradas semanalmente con la intención de cuantificar la motivación de los estudiantes. Estas primeras pruebas se llevaron a cabo durante las semanas sexta y décima del período académico; la aplicación se inició después del primer set de evaluación y se terminó poco después de la examinación del segundo set. 
Para el experimento, se buscó homogeneidad en ambos grupos (el de control y el experimental). Ambos grupos estaban a cargo del mismo profesor, recibían clases en el mismo salón, y estudiaban la misma temática (Electrónica). Sin embargo, los tamaños de las muestras no fueron iguales, ya que participaron 20 estudiantes en el grupo de control y 29 estudiantes para el grupo experimental; además, aunque todos los participantes estaban matriculados en el período enero-mayo de 2014, éstos podrían ir en distintos semestres, dado que la materia podría ser tomada en distintos momentos dentro de la carrera. En el grupo/escenario experimental, el profesor utilizaba, en cada clase, al menos tres prácticas propuestas por la neurociencia y mediadas por las tecnologías de información.

Se construyó un manual con las prácticas docentes que se decían aplicar en el grupo experimental. Dichas prácticas fueron tomadas de la literatura encontrada sobre el uso de los principios de las ciencias del cerebro aplicados en la educación y, en caso de ser posible, canalizadas a través de tecnologías de información (Bjork, mencionado en Sundem, 2012; Willis, 2009/2008/2006; Jensen, 2004; deWinstanley y Bjork, 2002). A continuación se menciona un breve resumen de estas actividades implementadas por el profesor durante el experimento: i) Realizar algún tipo de cambio en el patrón: cambiar los muebles, llevar un objeto diferente pero atractivo a la clase, caminar de otra manera, hacer pausas misteriosas; ii) Hacer una pregunta que demuestra un enlace de utilidad entre el curso y los planes futuros del estudiante; iii) Hacer una pregunta y explicar la relevancia de este tema, pero no dar la respuesta inmediatamente sino esperar unos minutos y luego dar la respuesta; iv) Después de un trabajo, y sin importar el resultado, dar su opinión sobre el esfuerzo del estudiante y no sobre los resultados, y recordar siempre hablarle por su nombre; v) Introducir el nuevo tema de la clase en términos de un problema conocido o una anécdota personal con el que los estudiantes pueden relacionarse; vi) Diseñar una actividad de clase que involucre desafío intelectual de por lo menos 5 minutos de duración con una recompensa previamente acordada por el grupo; vii) Si se está utilizando una presentación de PowerPoint, incluir preguntas con respuestas de opción múltiple y con imágenes que no estén directamente relacionados con el tema, de tal manera de establecer relaciones no intuitivas; viii) A mitad de la sesión, presentar un video multimedia que complemente los temas vistos en clase. El video debe ser fresco y con la mejor calidad disponible; ix) Cuando el contenido es teórico, durante la sesión se debe definir que los estudiantes solo pueden tomar los apuntes de clase en sus celulares. Las computadoras no están permitidas; y x) En otra sesión, hay que especificar que los estudiantes no pueden tomar notas durante la clase, sino que deben esperar hasta 50 10 minutos antes del final para tomarlas.

\section{RESULTADOS}

Después de examinar los datos obtenidos en las pruebas realizadas y las calificaciones de los estudiantes, se encontró que las tasas de atención, motivación y el rendimiento académico aumentaron en el grupo experimental (E) y no en el grupo de control (C) (ver Tabla 3). Después de la normalización de datos, el nivel de atención en el grupo experimental aumentó en un promedio de $12,5 \%$, mientras que en el grupo de control solo había un incremento del $4 \%$. El nivel de motivación observada en el grupo experimental aumentó en promedio un $11 \%$ y un $5 \%$ en la percepción reportada en la encuesta; mientras que en el grupo de control hubo un aumento de $9 \%$ en la motivación observada y una disminución de $5 \%$ en la percepción reportada. Por último, las puntuaciones globales (indicador de rendimiento académico) del grupo experimental fueron en promedio 13 de 100 puntos más altos, mientras que en el grupo de control el aumento fue de 7 puntos. Es importante resaltar que ambos grupos empezaron con una puntuación similar.

Tabla 3: Resultados de las pruebas.

\begin{tabular}{|c|c|c|c|}
\hline & $\begin{array}{c}\text { Experimental vs. Control } \\
\text { (Pre) }\end{array}$ & $\begin{array}{c}\text { Experimental vs. Control } \\
\text { (Post) }\end{array}$ & $\begin{array}{c}\text { Pre (E1) vs. Post (E2) } \\
\text { (Experimental) }\end{array}$ \\
\hline Atención & $\begin{array}{l}E(M=57.1, S D=25.4) \\
C(M=53.3, S D=28.3) \\
t=1.05, p=0.32\end{array}$ & $\begin{array}{l}E(M=68.9, S D=27.3) \\
C(M=56.2, S D=23.8) \\
t=2.27, p=0.04\end{array}$ & $\begin{array}{l}E 1(M=57.1, S D=25.4) \\
E 2(M=68.9, S D=27.3) \\
t=-2.79, p=0.02\end{array}$ \\
\hline $\begin{array}{l}\text { Motivación } \\
\text { observada }\end{array}$ & $\begin{array}{l}E(M=48.6, S D=33.0) \\
C(M=53.3, S D=31.3) \\
t=-1.20, p=0.26\end{array}$ & $\begin{array}{l}E(M=67.9, S D=27.8) \\
C(M=62.1, S D=28.9) \\
t=1.96, p=0.04\end{array}$ & $\begin{array}{l}E 1(M=48.6, S D=33.0) \\
E 2(M=67.9, S D=27.8) \\
t=-3.17, p=0.01\end{array}$ \\
\hline $\begin{array}{l}\text { Motivación } \\
\text { percibida }\end{array}$ & $\begin{array}{l}E(M=3.9, S D=0.2) \\
C(M=3.9, S D=0.3) \\
t=0.30, p=0.38\end{array}$ & $\begin{array}{l}E(M=4.2, S D=0.2) \\
C(M=3.7, S D=0.3) \\
t=3.48, p=0.00\end{array}$ & $\begin{array}{l}E 1(M=3.9, S D=0.2) \\
E 2(M=4.2, S D=0.2) \\
t=-2.66, p=0.01\end{array}$ \\
\hline $\begin{array}{l}\text { Desempeño } \\
\text { académico }\end{array}$ & $\begin{array}{l}E(M=46.8, S D=1.4) \\
C(M=46.0, S D=1.7) \\
t=1.6, p=0.12\end{array}$ & $\begin{array}{l}E(M=60.0, S D=1.2) \\
C(M=52.7, S D=1.7) \\
t=15.4, p=0.00\end{array}$ & $\begin{array}{l}E 1(M=46.8, S D=1.4) \\
E 2(M=60.0, S D=1.2) \\
t=-37.6, p=0.00\end{array}$ \\
\hline
\end{tabular}


Las variables analizadas en las pruebas presentadas en la Tabla 3 fueron: a) los niveles de atención y motivación observada, cuyos valores fueron expresados en términos de una escala normalizada del conteo de incidencias según la categoría de comportamiento; b) la motivación percibida por los estudiantes, expresada en una escala ascendente con valores de 1 a 5 y; c) las calificaciones individuales de los estudiantes del primer examen del curso y del examen final.

Para cada variable la estrategia de análisis consistió en tres fases: (1) Una prueba t independiente para evaluar si los grupos tenían valores homogéneos al inicio de las observaciones, es decir, definir si los grupos de control y experimental partían en condiciones semejantes de atención, motivación y rendimiento académico; (2) una prueba $t$ independiente para detectar diferencias entre los grupos en el escenario posterior de las observaciones; (3) una nueva prueba t, esta vez pareada, para evaluar si los alumnos del grupo experimental reportaron alguna variación significativa en términos de valores iniciales y finales de la intervención.

La prueba previa a la intervención, que midió tanto las variables del grupo experimental como del grupo de control, no fue significativa para ninguna de ellas. Es decir, ya que no se encontraron diferencias entre los valores, se entiende que ambos grupos tenían los mismos niveles de atención, motivación observada, motivación percibida y desempeño académico. Por otro lado, la prueba posterior a la intervención, que midió las mismas variables para el grupo experimental y contra el grupo de control, fue significativa para todas estas variables, siendo más altos los valores del grupo experimental. Esto permite reconocer que los valores del grupo experimental y el grupo de control parten de un mismo punto, pero se separan a lo largo de la intervención, terminando el grupo experimental con cifras más altas. Finalmente, a fin de confirmar los resultados, se corrió una prueba que midió específicamente los valores de previos y posteriores del grupo experimental. Los resultados muestran que los valores de las observaciones finales son más altos que los iniciales, confirmando así el cambio experimentado en el grupo intervenido.

\section{CONCLUSIONES}

El trabajo del profesor no es solo impartir una cátedra sino procurar el máximo aprendizaje de los alumnos. Tan importante es la capacidad del profesor para transmitir conocimiento sobre su materia, como su capacidad para captar la atención de sus alumnos, de motivarlos a aprender, e incluso de facilitarles la memorización. En los últimos años, las ciencias del cerebro han propuesto diferentes prácticas docentes que prometen facilitar estos procesos cognitivos básicos para el aprendizaje.

Los resultados de esta investigación muestran una asociación entre las prácticas docentes propuestas por la neurociencia, con el incremento de la atención, la motivación y el desempeño académico en los estudiantes de nivel universitario. Si bien el alcance del estudio fue descriptivo y exploratorio, los resultados de esta investigación alientan la ejecución de más investigaciones interdisciplinarias en la temática.

Un mejor entendimiento sobre el impacto de las ciencias del cerebro en la educación universitaria podría ser de mucha utilidad. Sin embargo, la interpretación de los resultados de este estudio debe ser analizada a la luz de las limitaciones del mismo. Para investigaciones futuras, se vislumbra considerar factores que puedan influir en los resultados como el nivel socioeconómico de los participantes, su situación laboral y/o condiciones familiares. Un mayor tamaño de la muestra estudiada, perfiles diversos de los sujetos de estudio y un mayor tiempo de exposición a las prácticas docentes, son también aspectos que siguientes estudios podrían considerar.

\section{REFERENCIAS}

Álava, C., Psicología de las emociones y actitudes: Lenguaje no verbal: gestos y ademanes, Alfaomega, México (2004)

Ansari, D., De Smedt, B. y Grabner, R., Neuroeducation: A critical overview of an emerging field, Neuroethics, 5(2), 105-117 (2012)

Arnett, J., Emerging adulthood: A theory of development from the late teens through the twenties, American Psychologist, 55(5), 469-480 (2000)

Beck, R., Taylor, C. y Robbins, M., Missing home: Sociotropy and autonomy and their relationship to psychological distress and homesickness in college freshmen, Anxiety, Stress and Coping, 16(2), 155-162 (2003)

Booth, J. y otros nueve autores, Neural development of selective attention and response inhibition, Neuroimage, 20(2), 737-751 (2003) 
Brickenkamp, R. y Zillmer, E., Test de atención d2, TEA Ediciones, Madrid, España (2002)

Bruning, R., Schraw, G., Norby, M. y Ronning, R., Psicología cognitiva y de la instrucción, Pearson-Prentice Hall, Madrid, España (2012)

Daw, N. y Shohamy, D., The cognitive neuroscience of motivation and learning, Social Cognition, 26(5), 593$620(2008)$

deWinstanley, P. y Bjork, R., Successful lecturing: Presenting information in ways that engage effective processing. En Halpern, D. y Hakel, M. (Eds.), Applying the Science of Learning to University Teaching and Beyond (pp. 19-31), Jossey-Bass, San Francisco, California (2002)

Dyson, R. y Renk, K., Freshmen adaptation to university life: Depressive symptoms, stress and coping, Journal of Clinical Psychology, 62(10), 1231-1244 (2006)

Fast, J., Body language, Lancaster Gazelle, New York, New York (2002)

Gazzaley, A. y Nobre, A., Top-down modulation: Bridging selective attention and working memory, doi: 10.1016/j.tics.2011.11.014, Trends in Cognitive Science, (en línea), 16(2), 129-135 (2012)

Glynn, S.M., Taasoobshirazi, G. y Brickman, P., Science motivation questionnaire: Construct validation with nonscience majors, Journal of Research in Science Teaching, 46(2), 127-146 (2009)

Haber, S.N. y Knutson, B., The reward circuit: Linking primate anatomy and human imaging, Neuropsychopharmacology, 35(1), 4-26 (2010)

Fischer, K. y Immordino-Yang, M., The Jossey-Bass reader on the brain and learning, Jossey-Bass, San Francisco, California (2008)

Jensen, E., Cerebro y aprendizaje. Competencias e implicaciones educativas, Narcea S.A. Ediciones, Madrid, España (2004)

van der Meulen, A., Krabbendam, L. y de Ruyter, D., Educational neuroscience: Its position, aims and expectations, doi: 10.1080/00071005.2015.1036836, British Journal of Educational Studies, (en línea), 63(2), 229-243 (2015)

Moscovitch, M., Nadel, L., Winocur, G., Gilboa, A. y Rosenbaum, R.S., The cognitive neuroscience of remote episodic, semantic and spatial memory, Current Opinion in Neurobiology, 16(2), 179-190 (2006)

Oliver, M., Towards an understanding of neuroscience for science educators, doi:

10.1080/03057267.2011.604478, Studies in Science Education, (en línea), 47(2), 211-235 (2011)

Peña-Casanova, J., Guardia J., Bertran-Serra I., Manero R.M. y Jarne, A., Versión abreviada del test Barcelona (I): Subtests y perfiles normales, Neurología 12(3), 99-111 (1997)

Pulido, R. y otros cinco autores, Estrés académico en estudiantes universitarios, Psicología y Salud, 21(1), 31-37 (2011)

Putwain, D., Researching academic stress and anxiety in students: Some methodological considerations, British Educational Research Journal, 33(2), 207-219 (2007)

Ribbens, G. y Thompson, R., Aprender las claves del lenguaje corporal en una semana, Gestión 2000, Barcelona, España (2002)

Riccio, D.C., Rabinowitz, V.C. y Axelrod, S., Memory: When less is more, American Psychologist, 49(11), 917-926 (1994)

Stevens, C. y Bavelier, D., The role of selective attention on academic foundations: A cognitive neuroscience perspective, Developmental Cognitive Neuroscience, 2S, S30-S48 (2012)

Sundem, G., Brain trust: 93 top scientists reveal lab-tested secrets to surfing, dating, dieting, gambling, growing man-eating plants, and more!, Three Rivers Press, New York, New York (2012)

van Kesteren M., Ruiter D., Fernández G, y Henson R., How schema and novelty augment memory formation, Trends in Neurosciences, 35(4), 211-219 (2012)

Willis, J., Research-based strategies to ignite student learning: Insights from a neurologist and classroom teacher, Alexandria, Va. Association for Supervision and Curriculum Development (2006)

Willis, J., Building a bridge from neuroscience to the classroom, doi: $10.1177 / 003172170808900608$, Phi Delta Kappan, (en línea), 89(6), 424-427 (2008)

Willis, J., What you should know about your brain, Educational Leadership, 67(4), 1-3 (2009) 
